INTERNATIONAL HIGHER EDUCATION Number 75 Spring 2014

Pages 8-10

\title{
Outcomes Assessment in International Education
}

\author{
DARLA K. DEARDORFF
}

Darla K. Deardorff is executive director of the Association of International Education Administrators and a research scholar at Duke University, Durham, North Carolina, US. E-mail: d.deardorff@duke.edu.

Due to the growing trend in higher education accountability, many postsecondary institutions are now measuring student learning outcomes, related to global or intercultural learning. However, a closer look is required at those assessment efforts, which although growing in popularity are not always designed well, executed effectively, or leveraged to maximum effect.

Often times, institutions engaged in outcomes assessment within international education will do the following: Have one person or one office "do the assessment"; use only one assessment tool (usually a pre/post tool); and use that particular tool because another university or all universities in a certain group are using it. Sometimes an institution will even design their own tool, often not vetting it for reliability or validity.

Far too often the assessment effort is an afterthought or an ad hoc effort, without sufficient work exerted at the planning stage, without clearly articulated goals and outcome statements, and without an assessment plan in place. Furthermore, the institution or program may simply shelve the data it has collected, claiming to have done assessment, ending the process there, and 
repeating this process again in subsequent years, as long as funding or staffing is available. The assessment data are rarely provided back to the students for their own continued learning and development that are crucial in intercultural learning. We outline several principles to ensure quality assurance in the student learning outcomes assessment practice in international education.

\section{A Road Map}

Higher education institutions embarking on assessment efforts will often start by asking, "Which tool should we use?" While this may seem like a logical place to start, it is important to first ask "What is it that we want to measure?" This question will lead to a closer examination of stated mission and goals that determine the appropriate assessment tools. When considering an assessment agenda for an international education program or initiative, it is helpful to step back and reflect on the following three questions, to help create an assessment road map: (1) Where are we going? (mission/goals); (2) How will we get there? (objectives/outcomes); and (3) How will we know when we have arrived? (evidence). Possibly, the evidence of student success goes beyond counting numbers (which are the outputs) to perceptions of students' learning (indirect evidence such as through surveys or inventories) and actual learning (direct evidence of student learning such as assignments in e-portfolios). This crucial alignment of mission, goals, and outcomes will naturally point to which tools/methods are needed to collect evidence that these outcomes have been achieved. 


\section{No Perfect Tool}

Assessment tools must be aligned with stated objectives and selected based on "fitness for purpose," rather than for reasons of convenience or familiarity. Too often, institutions or programs seek the one "perfect tool," which simply does not exist, especially for intercultural learning. In fact, when assessing something as complex as global learning or intercultural competence development, rigorous assessment involves the use of a multimethod, multiperspective approach that goes beyond the use of one tool. Furthermore, it is critical that institutions thoroughly explore existing tools in terms of exactly what those measure (not just what tools say they measure), the reliability and validity of the tools, the validity of the tool in that particular institutional/programmatic context, the theoretical basis of the tools, and including how well the tools align with the specific outcomes to be assessed. The prioritized outcomes will vary by the institution, so there is no one-size-fits-all approach when it comes to assessment tools.

As to decisions about assessment at preliminary ("pre") versus concluding ("post") stages of a program or course, good assessment means efforts are also ideally integrated into programming on an ongoing basis, avoiding the reliance on snapshots only at the beginning and/or end of a learning experience. Furthermore, the most meaningful and useful assessment of intercultural learning arguably contains a longitudinal component and provides feedback to students.

\section{Working from the Plan}

Another key principle of good assessment is that efforts need to be holistically developed and documented through an assessment plan. An assessment plan 
outlines not only what will be measured and how the data will be collected, but also details about who will be involved (which needs to be more than one person or office), the timeline, implementation details, and how the data will be used and communicated. This last point is crucial: there must be a use for the data (i.e., for student feedback, program improvement, and advocacy) or there is no need to collect the data. In particular, offices should not be collecting data and then trying to determine "what to do with it." Spending 10 percent of the time in the beginning to develop an assessment plan and thinking through these issues is time well invested in the later 90 percent of the effort that goes into assessment.

\section{A Team Effort}

Often, assessment can seem quite overwhelming and daunting, especially if only one person or office is tasked with doing it. Effective assessment actually involves an intrainstitutional team of stakeholders, which is comprised not only of international education experts but also assessment experts, students, faculty, and others who have a stake in international education outcomes. Senior leadership and support play a critical role in the success of assessment efforts. Once assembled, this intrainstitutional team prioritizes outcomes to be assessed, conducts an audit of assessment efforts already underway, and adapts current assessment efforts to align with goals and outcomes-no need to reinvent assessment efforts or add expensive ones when they may not be necessarybefore seeking additional assessment tools/methods that collect evidence needed to address stated goals and outcomes. 


\section{CONCLUSION}

There are other principles of effective assessment that might include utilizing a control group, best practices in terms of sampling, the use of longitudinal studies, and so on. This article has outlined a few principles as a call for further reflection and discussion on what truly makes for rigorous outcomes assessment in international education. While it is commendable for institutions to be engaged in outcomes assessment, it is important to take a closer look at the quality of the assessments being done. Guiding questions can include: How well are assessment tools/methods aligned with mission and goals? (Exactly what do those tools measure and why are they being used?) Is there more than one tool being used? Is there an assessment plan in place? How are assessment efforts integrated throughout a course or program, beyond pre/post efforts? How are the data being used? Is more than one person or office involved in assessment efforts? Is the assessment plan itself being reviewed regularly for improvement?

If higher education institutions are serious about internationalization, assessment, and student learning, such efforts are effective, resulting in outcomes that are meaningful for all involved, including our students. 